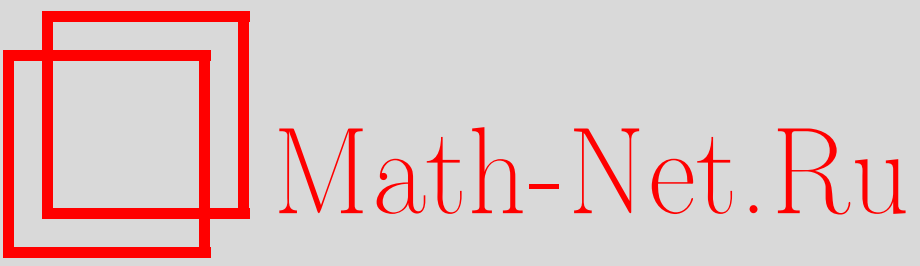

С. Б. Лебле, Необходимые условия ковариантности пары Лакса с одним полем, ТМФ, 2005, том 144, номер 1, 122-132

DOI: https://doi.org/10.4213/tmf1838

Использование Общероссийского математического портала Math-Net.Ru подразумевает, что вы прочитали и согласны с пользовательским соглашением

http://www.mathnet.ru/rus/agreement

Параметры загрузки:

IP: 18.207 .199 .55

26 апреля 2023 г., 07:37:37 
ТЕОРЕТИЧЕСКАЯ

И МАТЕМАТИЧЕСКАЯ

ФИЗИКА

Том 144, № 1

июль, 2005

(C) 2005 г.

С. Б. Лебле*

\section{НЕОБХОДИМЫЕ УСЛОВИЯ КОВАРИАНТНОСТИ ПАРЫ ЛАКСА С ОДНИМ ПОЛЕМ}

Изучается ковариантность по отношению к преобразованиям Дарбу полиномиальных дифференциальных и разностных операторов с коэффициентами, являющимися функциями одного базисного поля. В скалярном (абелевом) случае дифференциал $\Phi$ реше (первый член ряда Тейлора на пространстве продолжения) приравнивается к преобразованию Дарбу с целью установения функциональной зависимости; рассматривается пара Лакса для уравнения Буссинеска. Для пары обобщенных задач Захарова-Шабата (с операторами дифференцирования и сдвига) с операторными коэффициентами построен набор интегрируемых нелинейных уравнений вместе с явными формулами одевания. Неабелевы специальные функции выбираются как поля ковариантных пар. Вводятся разностная пара Лакса, комбинация преобразования Дарбу и калибровочного преобразования, а также решения уравнений Нама.

Ключевые слова: преобразование Дарбу, пара Лакса, уравнение Буссинеска, полиномы оператора сдвига, уравнение Нама.

\section{1. ВВЕДЕНИЕ}

Рассмотрим некоторый вид форминвариантностности дифференциальных многочленов с учетом связи между оператором

$$
\sum_{k=0}^{n} a_{k} \partial^{k}
$$

и оператором того же порядка, но с новыми (преобразованными) коэффициентами

$$
\sum_{k=0}^{n} a_{k}[1] \partial^{k}
$$

Будем называть это свойство “ковариантностью” оператора под действием преобразований некоторого вида. Подобный же термин будем использовать и в случае полиномиального оператора сдвига.

${ }^{*}$ Technical University of Gdan'sk, ul. G. Narutowicza, 11/12, 80-952, Gdan'sk-Wrzeszcz, Poland. E-mail: leble@mifgate.pg.gda.pl 
Доказательство ковариантности уравнения

$$
\psi_{t}=\sum_{k=0}^{n} a_{k} \partial^{k} \psi
$$

с некоммутативными коэффициентами $a_{k}$ по отношению к классическому преобразованию Дарбу (ПД) [1]

$$
\psi[1]=\psi^{\prime}-\sigma \psi
$$

включает вспомогательное соотношение (в статье используется сокращенное обозначение $\left.\psi^{\prime}=\partial \psi=\psi_{x}\right)[2]$

$$
\sigma_{t}=\partial r+[r, \sigma], \quad r=\sum_{0}^{N} a_{n} B_{n}(\sigma),
$$

где $B_{n}$ - дифференциальные полиномы Белла [3]. Соотношение (3) обобщает так называемое отображение Миуры и преврашается в тождество, когда $\sigma=\phi^{\prime} \phi^{-1}$, где $\phi-$ решение уравнения (1).

При изучении совместно ковариантных комбинаций (абстрактных) производных вводят дополнительные связи для полиномиальных коэффициентов, которые можно классифицировать [4], [5]. В двух словах, при наличии общего утверждения относительно ковариантого вида линейного полиномиального дифференциального оператора, который определяет формулы преобразования для коэффишиентов, самосогласованность между такими формулами приводит к появлению связей. В скалярном случае конструкции с одним потенциалом для уравнений Кортвега-де Фриза (КдФ) и Буссинеска изучались в работах [5], [6] (см. п. 2.1 данной работы) в применении к высшим уравнениям КдФ и Кадомцева-Петвиашвили (КП) [7].

Примеры нелокальных уравнений (с неабелевыми коэффициентами), интегрируемых методом ПД, рассматривались в работах [1], [8]. Некоторые из них, обзор которых приведен в книгах [9], [10], были недавно обобшены на широкий класс полиномов от автоморфизмов дифференциального кольца [11]. Исследование некоммутативных коэффициентов является более сложным, но и значительно более богатым и многообешающим. Это видно уже с точки зрения симметрийной классификации, изучение которой началось с работ [12] (см. также [13], [14] и обзор [15]). Далее, связь с подходом на основе ПД-ковариантности [16] позволяет построить и классифицировать ковариантные функции для использования их в квантовой [17] и солитонной задачах. Например, имеется класс уравнений вида

$$
-i \rho_{t}=[H, h(\rho)]
$$

где $h(\rho)$ - аналитическая функция. В квантовой механике операторы $\rho$ и $H$ играют роли матрицы плотности и гамильтониана. Ковариантность уравнений (4) по отношению к ПД установлена в работе [18]. Случаи $f(\rho)=i \rho^{3}$ и $f(\rho)=i \rho^{-1}$ были рассмотрены в связи с приложениями в работе [19]. Следующий шаг к сушественно неабелевым функциям, например

$$
h(X)=X A+A X
$$


$[A, X] \neq 0$, восходит к работам [20], а обобщения изучаются в статье [16]. Дальнейшие обобщения представлены в работе [21], где приведен богатый список интегрируемых уравнений. Этот список частично соответствует результатам работы [13], причем установлена также обычная для техники ПД связь с решениями посредством итерационных процедур или одевающих цепочек.

\section{2. ПАРА ЛАКСА С ОДНИМ ПОЛЕМ ДЛЯ АБЕЛЕВА СЛУЧАЯ}

2.1. Уравнения ковариантности. Сначала, как обобшение исследований примера уравнения Буссинеска [5], [22], воспроизведем схему с одним коммутативным скалярным полем. Рассмотрим уравнение (1) с коэффициентами $b_{k}, k=0,1,2,3$, а коэффициенты $a_{k}$ будем использовать для второго оператора в паре Лакса. ПД для операторных коэффициентов третьего порядка (обобшение Матвеева классического П Д [1]) приводит к соотношениям

$$
\begin{aligned}
& b_{2}[1]=b_{2}+b_{3}^{\prime}, \quad b_{1}[1]=b_{1}+b_{2}^{\prime}+3 b_{3} \sigma^{\prime}, \\
& b_{0}[1]=b_{0}+b_{1}^{\prime}+\sigma b_{2}^{\prime}+3 b_{3}\left(\sigma \sigma^{\prime}+\sigma^{\prime \prime}\right),
\end{aligned}
$$

причем $b_{3}$ не преобразуется. Предположим, что оба оператора зависят от единственной потенциальной функции $w$. Задача для первого оператора формулируется следующим образом: найти ограничения на коэффициенты $b_{3}(x, t), b_{2}(x, t), b_{1}=b(w, t), b_{0}=$ $G(w, t)$, совместные с правилами ПД (6), (7) для потеншиальной функции $w$, индушированной ПД для $b_{1}$ или $b_{0}$. Для простоты положим $b_{3}^{\prime}=0$.

Ковариантность соответствуюшего спектрального уравнения

$$
b_{3} \psi_{x x x}+b_{2}(x, t) \psi_{x x}+b(w, t) \psi_{x}+G(w, t) \psi=\lambda \psi
$$

приводит к связи только между коэффициентами $b_{1}$ и $b_{0}$. При исследовании задачи (8) в контексте представления Лакса для некоторого нелинейного уравнения ковариантность второго уравнения Лакса следует учесть с самого начала. Назовем такой принцип “принципом совместной ковариантности" [4]. Форма второго уравнения Лакса фиксирует положение поля $w$ :

$$
\psi_{t}=a_{2}(t) \psi_{x x}+a_{1}(t) \psi_{x}+w \psi
$$

Если рассмотреть уравнения (8), (9) как уравнения для пары Лакса, ПД функции $w$ должно быть совместно с формулами ПД (подобными (6)) для обоих коэффициентов из уравнения (8), зависящих от единственной переменной $w$. Далее, для задачи (9) обобщенное отображение Миуры (3) имеет вид [2]

$$
\sigma_{t}=\left[a_{2}\left(\sigma^{2}+\sigma_{x}\right)+a_{1} \sigma+w\right]_{x}
$$

а для спектрального уравнения (8) -

$$
b_{3}\left(\sigma^{3}+3 \sigma_{x} \sigma+\sigma_{x x}\right)+b_{2}\left(\sigma^{2}+\sigma_{x}\right)+b(w, t) \sigma+G(w)=\mathrm{const} ;
$$


здесь $\phi$ является решением обоих уравнений Лакса.

Предположим далее, что коэффициенты операторов являются аналитическими функциями от $w$ вместе со своими производными (или интегралами) по $x$ (такие функции носят название функций на пространстве продолжения). Для коэффициента $b_{1}=b\left(\partial^{-1} w, w, w_{x}, \ldots, \partial^{-1} w_{t}, w_{t}, w_{t x}, \ldots\right)$ возникает условие ковариантности для дифференциала Фреше (ДФ) функции $b$ на пространстве продолжения. Приравнивая это разложение и ПД, получаем условие

$$
b_{2}^{\prime}+3 b_{3} \sigma^{\prime}=b_{w}\left(a_{1}^{\prime}+2 a_{2} \sigma^{\prime}+\sigma a_{2}^{\prime}\right)+b_{w^{\prime}}\left(a_{1}^{\prime}+2 a_{2} \sigma^{\prime}+\sigma a_{2}^{\prime}\right)^{\prime}+\cdots
$$

Мы называем это уравнение (первым) “уравнением совместной ковариантности”. Оно гарантирует совместность коэффициентов преобразований пары Лакса (8), (9). Сравнение двух преобразований дает следуюшее выражение для $b(w, t)($ с произвольным $\alpha(t))$ :

$$
b(w, t)=\frac{3 b_{3} w}{2 a_{2}}+\alpha(t) .
$$

Приравнивая разложение $b_{0}=G(\ldots, w, \ldots)$ на пространстве продолжения

$$
G\left(w+a_{1}^{\prime}+2 a_{2} \sigma^{\prime}+\sigma a_{2}^{\prime}\right)=G(w)+G_{w}\left(a_{1}^{\prime}+2 a_{2} \sigma^{\prime}+\sigma a_{2}^{\prime}\right)+\cdots
$$

и ПД (7) того же коэффициента, получаем

$$
\begin{aligned}
b_{1}^{\prime} & +\sigma b_{2}^{\prime}+3 b_{3}\left(\frac{\sigma^{2}}{2}+\sigma^{\prime}\right)^{\prime}= \\
& =G_{w_{x}}\left(a_{1}^{\prime}+2 a_{2} \sigma^{\prime}+\sigma a_{2}^{\prime}\right)^{\prime}+G_{\partial^{-1} w_{t}}\left[a_{1 t}+2 \partial^{-1}\left(a_{2} \sigma_{t}^{\prime}\right)+\partial^{-1}\left(\sigma a_{2}^{\prime}\right)_{t}\right]+\cdots
\end{aligned}
$$

Это второе "уравнение совместной ковариантности” также упрошается при $a_{2}^{\prime}=0$. Заметим, что соотношение (10), использованное в левой части (15), линеаризует ДФ по $\sigma$. Окончательно получим

$$
\begin{gathered}
b_{2}=\frac{3 b_{3} a_{1}}{2 a_{2}}+\beta(t), \\
G(w, t)=\frac{3 b_{3} w_{x}}{2 a_{2}}+\frac{3 b_{3} a_{1}^{\prime} \partial^{-1} w}{2 a_{2}^{2}}+\frac{3 b_{3} \partial^{-1} w_{t}}{2 a_{2}^{2}} .
\end{gathered}
$$

ЗАмЕчАниЕ 2.1. Мы ограничили формулы для ДФ уровнем, необходимым для минимальных потоков. Учет высших членов приводит ко всей иерархии, как в работе [7] для случая $\mathrm{K}$ д $\Phi-\mathrm{KП.}$ 
2.2. Условия совместности. В случае, когда $a_{2}^{\prime}=0$, которым мы ограничились, система Лакса (8), (9) приводит к условиям совместности

$$
\begin{aligned}
& b_{3 t}=2 a_{2} b_{2}^{\prime}-3 b_{3} a_{1}^{\prime \prime}, \\
& b_{2 t}=a_{2} b_{2}^{\prime \prime}+2 a_{2} b_{1}^{\prime}+a_{1} b_{2}^{\prime}-3 b_{3} a_{1}^{\prime \prime}-2 b_{2} a_{1}^{\prime}-3 b_{3} a_{0}^{\prime}, \\
& b_{1 t}=a_{2} b_{1}^{\prime \prime}+a_{1} b_{1}^{\prime}-b_{3} a_{1}^{\prime \prime \prime}-b_{2} a_{1}^{\prime \prime}-b_{1} a_{1}^{\prime}-3 b_{3} a_{0}^{\prime \prime}-2 b_{2} a_{0}^{\prime}+2 a_{2} b_{0}^{\prime}, \\
& b_{0 t}=a_{1} b_{0}^{\prime}+a_{2} b_{0}^{\prime \prime}-b_{1} a_{0}^{\prime}-b_{2} a_{0}^{\prime \prime}-b_{3} a_{0}^{\prime \prime \prime} .
\end{aligned}
$$

Связи $(16),(17)$ в комбинации с выражением для $b_{2 t}$ дают

$$
\beta_{t}=-2 \beta a_{1}^{\prime} \text {. }
$$

Два последних уравнения (с константами, выбранными как $b_{3}=1, a_{2}=-1, \quad b_{2}^{\prime}=$ $\left.a_{1}^{\prime}=0\right)$ дают

$$
\frac{3 b_{3}\left(w_{t}+a_{1} w\right)_{t}}{4 a_{2}^{2}}=-\left[\left(\frac{3 b_{3} w}{2 a_{2}}+\alpha\right) w^{\prime}-\frac{b_{3} w^{\prime \prime \prime}}{4}+\frac{3 b_{3} a_{1} w_{t}}{4 a_{2}^{2}}+\left(\beta-\frac{3 b_{3} a_{1}}{4 a_{2}}\right) w^{\prime \prime}\right]^{\prime}
$$

Это уравнение редуцируется к уравнению Буссинеска (см., например, [9]) при $b_{1}=a_{1}=$ $0, b_{3}=1, a_{2}=-1$.

\section{3. НЕАБЕЛЕВ СЛУЧАЙ. ЗАДАЧА ЗАХАРОВА-ШАБАТА}

3.1. Условия совместной ковариантности для общих уравнений Захарова-Шабата (ЗШ). Для уравнения (1) первого порядка $(n=1)$ с коэффициентами из неабелева дифференциального кольца $A$ (подробности см. в работе [2]) изменим обозначения следуюшим образом:

$$
\psi_{t}=(J+u \partial) \psi
$$

где оператор $J \in A$ не зависит от $x, y, t$, а потенциал $a_{0} \equiv u=u(x, y, t) \in A$ является функцией всех переменных. Преобразованный потенциал имеет вид

$$
\tilde{u}=u+[J, \sigma]
$$

где $\sigma=\phi_{x} \phi^{-1}$, а $\phi \in A$ снова является решением уравнения (20).

Предположим, что второй оператор пары Лакса имеет тот же вид, но с другими входяшими в него элементами:

$$
\psi_{y}=(Y+w \partial) \psi
$$

где $Y \in A$ является постоянным элементом, а потенциал $w=F(u) \in A$ - функция потенциала первого уравнения (20). Тем самым принцип совместной ковариантности [4] принимает вид

$$
\widetilde{w}=w+[Y, \sigma]=F(u+[J, \sigma]),
$$

откуда непосредственно вытекает, что

$$
F(u)+[Y, \sigma]=F(u+[J, \sigma]) .
$$

Таким образом, уравнение (24) определяет функцию $F(u)$; мы по-прежнему называем это уравнение уравнением совместной ковариантности. 
3.2. Ковариантные комбинации симметрических многочленов. Первым естественным примером является обобшение уравнения волчка Эйлера с гамильтонианом (5), упомянутое во введении. Ковариантная пара Лакса в этом случае состоит из двух уравнений (20), (22), элементы операторов удовлетворяют “уравнению совместной ковариантности" (24) и условиям совместности, если $J=H, Y=H^{2}[16]$. Более обшая связь $Y=J^{n}, J=H$ приводит к ковариантности функции

$$
P_{n}(H, u)=\sum_{p=0}^{n} H^{n-p} u H^{p},
$$

приведенной в работе [16]. Для дальнейшего обобшения рассмотрим комбинации таких полиномов вида

$$
f(H, u)=H u+u H+S^{2} u+S u S+u S^{2} .
$$

Подставим $F(u)=f(H, u)$ вида (25) в (24); такая подстановка подразумевает выбор $Y=A B+C D E$, что приводит к соотношению

$$
\begin{gathered}
A[B, \sigma]+[A, \sigma] B+C D[E, \sigma]+C[D, \sigma] E+[C, \sigma] D E= \\
=H[J, \sigma]+[J, \sigma] H+S^{2}[J, \sigma]+S[J, \sigma] S+[J, \sigma] S^{2} .
\end{gathered}
$$

Это равенство выполняется тождественно, если $A=B=J=H, C=D=E=\alpha H$, $S=\beta H$, а также $[\alpha, H]=0,[\beta, H]=0$, с учетом связи $\alpha^{3}=\beta^{2}$. Продолжая такой анализ, приходим к следуюшему утверждению.

УТВЕРЖДЕНИЕ. Принцип совместной ковариантности определяет класс однородных полиномов $P_{n}(H, u)$, симметричных по отношению $к$ ииклическим перестановкам, как возможных гамильтонианов $h(u)=P_{n}(H, u)$ для әволюиии типа Лиувилля-фон Неймана (4). Линейная комбиначия таких полиномов $\sum_{n=1}^{N} \beta_{n} P_{n}(H, u)$ с коэффициентами, коммутирующими с и и $H$, также приводит к ковариантной паре, если выполнено соотношение $Y=\sum_{n=1}^{N} \alpha_{n} H^{n+1} u$ $\alpha_{1}=\beta_{1}=1, \quad \alpha_{n}^{n+1}=\beta_{n}^{n}, \quad n \neq 1$.

Доказательство можно провести по индукции, основанной на однородности $P_{n}$ и линейности связей по $u$. Функции $F_{H}(u)=\sum_{0}^{\infty} a_{n} P_{n}(H, u)$ также удовлетворяют связям и определяют некую функцию, если ряд сходится.

\section{4. НЕЛОКАЛЬНЫЕ ОПЕРАТОРЫ}

Пусть $A$ - операторное кольцо и для заданных элементов $f, g \in A$ автоморфизм $T$ обладает свойством $T(f g)=T(f) T(g)$. Общие формулы ПД для полиномов от оператора $T$ приведены в работе [11]. Будем называть оператор $T$ оператором сдвига, но, согласно данному определению, он может быть обшим. 
4.1. Эволюция оператора сдвига первого порядка с одним полем. Возьмем общее эволюционное уравнение в случае $N=1$ :

$$
\psi_{t}(x, t)=\left(U_{0}+U_{1} T\right) \psi .
$$

В этом случае имеются два типа ПД [11], [23], обозначаемые индексами “+” и “-”. ПД первого типа $(+)$ оставляет $U_{0}$ неизменным. Перепишем преобразование для $U_{1}$ в виде

$$
U_{1}^{+}=\sigma^{+}\left(T U_{1}\right)\left(T \sigma^{+}\right)^{-1},
$$

где $\sigma^{+}=\phi(T \phi)^{-1}$, далее верхний индекс “+” не указывается.

Нетривиальные уравнения цепочки появляются для стационарного варианта уравнения (26)

$$
(J+U T) \varphi=\varphi \mu,
$$

если постоянный элемент $\mu$ не коммутирует с $\varphi$ и $\sigma$.

Выведем тождество, связывающее потенциал $U$ и $\sigma$ (ср. [23]). Подставим соотношение

$$
T(\sigma) T^{2}(\varphi)=T(\varphi)
$$

в уравнение сдвига (28). Имеем связь типа отображения Миуры

$$
\sigma T(U) \sigma=U+[J, \sigma],
$$

где учтено, что $T(\sigma)=\sigma^{-1}$. Эта связь также упрощает формулы ПД (27):

$$
U^{+}=\sigma(T U) \sigma=U+[J, \sigma] .
$$

Прямое использование уравнения (28) для выражения $U$ через $\tau=\varphi \mu \varphi^{-1}$ и $\sigma$ дает $U=$ $\tau \sigma-J \sigma$. Подстановка результатов в (30) приводит к соотношению

$$
T(\tau)=\sigma^{-1} \tau \sigma,
$$

которое определяет элемент $\tau$ на множестве точек $T^{m} x_{0}$ как явную функцию $\sigma$ :

$$
\tau=\sigma^{-m} \tau\left(x_{0}\right) \sigma^{m} .
$$

Отсюда, в свою очередь, при использовании (33) получается явное выражение для $U$. Окончательно, подставляя $U$ в $(31)$, для всех $n, n+1$ получаем уравнение одеваюшей цепочки

$$
\sigma_{n}^{-m} \tau\left(x_{0}\right) \sigma_{n}^{m} \sigma_{n}-\sigma_{n} J=\sigma_{n+1}^{-m} \tau\left(x_{0}\right) \sigma_{n+1}^{m} \sigma_{n+1}-J \sigma_{n+1},
$$

параметризованное посредством $\mu$ через $\tau\left(x_{0}\right)$.

Уравнение цепочки является естественным результатом выражения ПД в терминах переменной $\sigma$ с помошью отображения Миуры. Оно изучалось в работе [24] в связи со знаменитой скалярной задачей КдФ и стандартнной задачей Штурма-Лиувилля. Периодическое замыкание цепочек дает интегрируемые бигамильтоновы конечномерные системы, а в некоторых специальных случаях - конечнозонные потенциалы [25]. Случай уравнения Буссинеска исследован в работе [22] (см. также [26]). Уравнения цепочки для классической дифференциальной задачи ЗШ и двух типов ПД были введены в работе [27] (см. также недавний обзор [15]). 
4.2. Уравнения совместной ковариантности. Возьмем реплику уравнения (26)

$$
\psi_{t}(x, t)=\left(V_{0}+V_{1}(U) T\right) \psi
$$

с заменой $U_{1} \rightarrow V_{1}=F(U)$. Тем самым вводится пара Лакса с одним полем, составленная из двух эволюционных уравнений ЗШ. Имеется в виду, что коэффициенты обоих уравнений зависят от единственного оператора $U$. Инвариантность постоянной $V_{0}$ следует как свойство ПД первого типа. Преобразуем $V_{1}$ с помошью (27), рассматривая результат как ту же самую функцию от $U_{1}^{+}$. Это приводит к уравнению совместной ковариантности

$$
V_{1}^{+}=F\left(U^{+}\right)=F\left(\sigma^{+}(T U)\left(T \sigma^{+}\right)^{-1}\right)=\sigma^{+}(T F(U))\left(T \sigma^{+}\right)^{-1}
$$

Аналогичное уравнение выводится для альтернативной “-”-системы.

\section{5. ТЕОРЕМЫ КОВАРИАНТНОСТИ ДЛЯ ВЫСШИХ ОПЕРАТОРОВ}

Следуюшая теорема устанавливает ковариантность обоих уравнений, порождаюших пару Лакса для уравнения Нама [28].

Tеорема 5.1. Уравнение

$$
\psi_{y}=u T \psi+v \psi+w T^{-1} \psi
$$

ковариантно по отночению к ПД, скомбинированному с калибровочнбц преобразованием

$$
\psi[1]=g(y)(T-\sigma) \psi,
$$

где $g(y) \in A, \sigma=(T \phi) / \phi, \quad \phi-(\partial р у г о е)$ решение того же уравнения (37). Преобразования коэффициентов уравнения имеют вид

$$
\begin{aligned}
u[1] & =g T(u)[T(g)]^{-1}, \\
v[1] & =g T(v) g^{-1}-g \sigma u g^{-1}+g T(u) T(\sigma) g^{-1}+g_{y} g^{-1}, \\
w[1] & =g \sigma w\left[T^{-1}(g \sigma)\right]^{-1} .
\end{aligned}
$$

ДоКАЗАТЕЛЬСтво. Подстановка (38) в преобразованное уравнение (37) дает четыре уравнения, причем $T^{n} \psi$ являются независимыми. Три из них приводят к преобразованным потеншиалам (39). Четвертое уравнение после применения преобразования принимает вид

$$
\sigma_{y}=\sigma F-(T F) \sigma, \quad F=u \sigma+v+w\left[T^{-1}(\sigma)\right]^{-1} .
$$

Можно проверить справедливость этого уравнения, подставляя определение $\sigma$ и используя уравнение для $\phi$.

5 Теоретическая и математическая физика, т. 144, № 1, 2005 г. 
ЗАмЕчАниЕ 5.1. Теорема 5.1 естественным образом справедлива для спектральной задачи

$$
\lambda \psi=u T \psi+v \psi+w T^{-1} \psi,
$$

которая возникает для стационарных решений уравнения (37) с единственным отсутствующим последним слагаемым для преобразования $v[1]$. Преобразование использует собственную функцию $\phi$ с отличным собственным значением $\mu$. Уравнение (40) переходит в соответствуюший преобразованию Миуры аналог для функции $\sigma$ :

$$
\mu=u \sigma+v+w\left[T^{-1}(\sigma)\right]^{-1} .
$$

\section{6. РЕДУКЦИИ УРАВНЕНИЯ НАМА}

Уравнение Нама можно записать в представлении Лакса (в пионерских работах [28] операторы пары Лакса - дифференциальные операторы), используя спектральное уравнение (41) и эволюционное уравнение

$$
\psi_{t}=(q+p T) \psi
$$

Ковариантность этого уравнения по отношению к ПД (38) можно установить подобно теореме 5.1 с учетом эволюции для функции $\sigma(t)$,

$$
\sigma_{t}=T(q) \sigma-\sigma p \sigma+T(p) T(\sigma) \sigma-\sigma q,
$$

что доказывает следуюшие формулы преобразования для коэффициентов в уравнении (43):

$$
\begin{aligned}
& p[1]=g T(p)[T(g)]^{-1}, \\
& q[1]=g[T(q)-\sigma p+T(p) T(\sigma)] g^{-1}+g_{y} g^{-1} .
\end{aligned}
$$

Выбор $p=u+\beta I, q=v / 2, T\left(\varphi_{i}\right)=\varphi_{i}$,

$$
u=\alpha\left(-\frac{\imath \varphi_{1}}{2}-\varphi_{3}\right), \quad v=\varphi_{3}, \quad w=\alpha^{-1}\left(-\frac{\imath \varphi_{1}}{2}+\varphi_{3}\right)
$$

приводит к уравнениям Нама

$$
\varphi_{i, t}=\imath \epsilon_{i k l}\left[\varphi_{k}, \varphi_{l}\right]
$$

Система (41), (43) ковариантна по отношению к комбинированному ПД и калибровочному преобразованию, если переменная $g=e^{G}$ выбрана так, что

$$
G_{t}=\frac{\alpha}{2}\left[T\left(\varphi_{3}+\frac{\varphi_{1}}{2}\right) T(\sigma)-\sigma\left(\varphi_{3}+\frac{\varphi_{1}}{2}\right)\right] .
$$

Наконец, можно сформулировать следуюшую теорему.

Теорема 6.1. Система (48) инвариантна по отношению $x$ преобразованиям

$$
\begin{aligned}
& \varphi_{1}[1]=g\left[\left(\frac{T\left(\varphi_{1}\right)}{2}-\imath T\left(\varphi_{3}\right)\right) T(g)^{-1}+\sigma\left(\frac{\varphi_{1}}{2}+\imath \varphi_{3}\right)\left[T^{-1}(g \sigma)\right]^{-1}\right], \\
& \varphi_{2}[1]=g\left[T\left(\varphi_{2}\right)+\alpha\left(\frac{\imath \sigma \varphi_{1}}{2}-\frac{\imath \varphi_{1} T(\sigma)}{2}+\sigma \varphi_{3}-T\left(\varphi_{3} \sigma\right)\right)\right] g^{-1}, \\
& \varphi_{3}[1]=g\left[\left(T\left(-\frac{\imath \varphi_{1}}{2}-\varphi_{3}\right)\right) T(g)^{-1}+\sigma\left(-\frac{\imath \varphi_{1}}{2}+\varphi_{3}\right)\left[T^{-1}(g \sigma)\right]^{-1}\right]
\end{aligned}
$$

с функиией $g=e^{G}$, әде $G$ получена интегрированием (49). 


\section{7. РЕШЕНИЯ УРАВНЕНИЯ НАМА}

Следуя построению, описанному в предыдушем разделе, проследим за алгоритмом на простом примере, рассматривая $T$ как оператор сдвига $T \psi(x, y)=\psi(x+1, y)$. В качестве затравочного решения уравнений Нама (48) рассмотрим коммутирующие постоянные матришы $\varphi_{i}=A_{i}, i=1,2,3$, что означает, что в уравнении (47) величины $u, v, w$ являются постоянными. Прежде всего следует построить решение пары Лакса $(41),(43)$, которое можно найти в виде (все элементы предполагаются обратимыми)

$$
\phi=\xi(t) \Phi(x) .
$$

Уравнение для $\xi$ получается в виде

и имеет решение

$$
\xi_{t}=\left[\frac{v}{2}+(u+\beta I) T\right] \xi=Z \xi
$$

$$
\xi=e^{Z t} \xi_{0}
$$

Подстановка $\phi$ в (41) приводит к спектральной задаче с разностными операторами сдвига:

$$
\mu \Phi(x)=\xi^{-1}[u \xi \Phi(x+1)+v \xi \Phi+w \xi \Phi(x-1)] .
$$

Снова разделяя переменные, строим класс частных решений в виде

$$
\Phi=\eta e^{\Sigma x}
$$

и таким образом получаем матричную спектральную задачу для $\eta$ :

$$
\mu \eta=\xi^{-1}\left[u \xi \eta e^{\Sigma}+v \xi \eta+w \xi \eta e^{-\Sigma}\right],
$$

с оператором в правой части, а потому со спектральным параметром $\mu$, параметризованным посредством $t$. Окончательно матрица $\sigma$ составляется как

$$
\sigma=\xi(t) \eta e^{\Sigma} \eta^{-1} \xi^{-1}(t) .
$$

Подходящий выбор коммутаторной алгебры для $A_{i}, \Sigma, \eta$ позволяет получить явньй вид $\sigma$ и, следовательно, построить и решить уравнение (49) для $G$ :

$$
G_{t}=\frac{\alpha}{2}\left[\left(\varphi_{3}+\frac{\varphi_{1}}{2}\right) \xi(t) \eta e^{\Sigma} \eta^{-1} \xi^{-1}(t)-\xi(t) \eta e^{\Sigma} \eta^{-1} \xi^{-1}(t)\left(\varphi_{3}+\frac{\varphi_{1}}{2}\right)\right],
$$

и, далее, ее экспоненту, т.е. матрицу $g$, необходимую для формул одевания (50). Подчеркнем, что матрицы $\sigma$ и $g$ не зависят от $x$, а потому от $x$ не зависит и одетая матрица $\varphi[i]$.

\section{8. ЗАКЛЮЧЕНИЕ}

В данной работе развит общий метод построения операторов с одним полем, ковариантных относительно ПД. С помощью этого метода можно получить уравнения совместной ковариантности для операторных коэффициентов как функций на пространстве продолжения. Найдены некоторые решения уравнений на пути обобшения тейлорова разложения функций [29].

Благодарности. Работа выполнена при частичной поддержке Министерства научных исследований и информационных технологий Польши, грант PBZ-Min-008/P03/03. 


\section{Список литературы}

[1] V. B. Matveev. Lett. Math. Phys. 1979. V. 3. P. 213, 217, 503.

[2] A. A. Zaitsev, S. B. Leble. Rep. Math. Phys. 2000. V. 46. P. 165; math-ph/9903005.

[3] F. di Bruno. Pure Appl. Math. 1857. V. 1. P. 359.

[4] S. Leble. Darboux transforms algebras in $2+1$ dimensions. In: Nonlinear Evolution Equations and Dynamical Systems. Proc. of the 7th Workshop (NEEDS-91) (Baia, Verde, Italy, June 19-29, 1991). Eds. M. Boiti, L. Martina, F. Pempinelli. Singapore: World Scientific, 1992. P. 53.

[5] С. Б. Лебле. ТМф. 2001. Т. 128. С. 65.

[6] F. Lambert, S. Leble, J. Springael. Glasgow Math. J. A. 2001. V. 43. P. 55.

[7] F. Lambert, I. Loris, J. Springael. Inverse Problems. 2001. V. 17. P. 1067.

[8] М. А. Салль. ТМФ. 1982. Т. 53. С. 227; С. Б. Лебле, М. А. Салль. ДАН СССР. 1985. T. 284. C. 110.

[9] V. B. Matveev, M. A. Salle. Darboux transformations and solitons. Berlin: Springer, 1991.

[10] C. Roger, W.K. Schiff. Backlund and Darboux Transformations. Cambridge: Camb. Univ. Press, 2002.

[11] V. B. Matveev. Am. Math. Soc. Transl. II. 2000. V. 201. P. 179.

[12] P. J. Olver, V. V. Sokolov. Commun. Math. Phys. 1998. V. 193. № 2. P. 245; Inverse Problems. 1998. V. 14. P. L5.

[13] А. В. Михайлов, В. В. Соколов. ТМФ. 2000. Т. 122. С. 88.

[14] R. H. Heredero, A. Shabat, V. Sokolov. J. Phys. A. 2003. V. 36. № 47. P. L605.

[15] А. Б. Шабат. ТМФ. 2003. Т. 136. № 2. С. 197.

[16] S. B. Leble, M. Czachor. Phys. Rev. E. 1998. V. 58. P. 7091.

[17] A. A. Andrianov, F. Cannata, M. V. Ioffe, D. N. Nishnianidze. J. Phys. A. 1997. V. 30. P. 5037.

[18] N. Ustinov, S. Leble, M. Czachor, M. Kuna. Phys. Lett. A. 2001. V. 279. P. 333.

[19] A. Minic. Phys. Lett. B. 2002. V. 536. P. 305.

[20] C. В. Манаков. Функц. анализ и его прилож. 1976. Т. 10. С. 93; M. Adler, P. van Moerbeke. Adv. Math. 1980. V. 38. P. 267.

[21] J. Cieslinski, M. Czachor, N. Ustinov. J. Math. Phys. 2003. V. 44. P. 1763.

[22] S. Leble. Covariant forms of Lax one-field operators: from Abelian to non-commutative. In: Bilinear Integrable Systems: from Classical to Quantum, from Continuous to Discrete. Proc. NATO Advanced Research Workshop (St.Petersburg, Russia, 15-19 September, 2002). NATO Sci. Ser. II: Math. Phys. Chem. V. 201. Eds. L. Faddeev, P. van Moerbeke, F. Lambert. Dordrecht: Kluwer, 2005; math-ph/0302053.

[23] S. Leble. Dressing chain equations associated with difference soliton systems. In: Probing the structure of quantum mechanics. Nonlinearity, nonlocality, computation, axiomatics. Eds. D. Aerts, M. Czachor, T. Durt. London: World Scientific, 2002. P. 354.

[24] J. Weiss. J. Math. Phys. 1986. V. 27. P. 2647.

[25] А. П. Веселов, А. Б. Шабат. Функц. анализ и его прилож. 1993. Т. 27. С. 1.

[26] В. Э. Адлер, В. Г. Марихин, А. Б. Шабат. ТМФ. 2001. Т. 129. № 2. С. 163.

[27] А. Б. IШабат. ТМФ. 1999. Т. 121. № 1. С. 165.

[28] W. Nahm. Phys. Lett. B. 1980. V. 90. P. 413; N. J. Hitchin. Commun. Math. Phys. 1983. V. 89. № 2. P. 145 .

[29] R. W. Atherton, G. M. Homsy. Studies Appl. Math. 1975. V. 54. P. 31. 